\title{
Selling less information for more: garbling with benefits
}

\author{
Thomas A. Weber ${ }^{\mathrm{a}, *}$, David C. Croson ${ }^{\mathrm{b}}$ \\ ${ }^{a}$ Department of Management Science and Engineering, Terman Engineering Center, Stanford University, \\ Stanford, CA 94305-4026, USA \\ ${ }^{\mathrm{b}}$ Department of Management Science, MIT Sloan School of Management E53-311; 30 Wadsworth Street, \\ Cambridge, MA 02140-1830, USA
}

Received 30 July 2002; received in revised form 8 October 2003; accepted 4 November 2003

\begin{abstract}
The expected value of information in a standard portfolio investment problem with ex-post payment can increase when the information is garbled prior to its sale. Distorting the information helps to resolve the incentive problem decreasing the buyer's default risk and thereby increasing the seller's expected revenues.
\end{abstract}

(C) 2004 Elsevier B.V. All rights reserved.

Keywords: Value of information; Portfolio investment; Limited liability; Garbling

JEL classification: C44; D81

\section{Introduction}

In decision problems where an agent's payoff depends on an exogenous random event, an informative signal about this event is valuable if it permits the agent to adjust her actions. The more informative the signal is in the sense of statistical sufficiency, the more valuable it is to an agent who uses it directly (Blackwell, 1953). We consider situations where an agent sells information to an investor and shows that the expected revenues from the sale may be non-monotonic in its informativeness. As an example we consider the sale of information in a simple portfolio investment problem, where the payment for the information is made after the return is realized, subject to limited liability. In the interesting case where no upfront payments for the information are permitted, we show that garbling the information (Marschak and Miyasawa, 1968) can strictly increase expected revenues for the seller by influencing the investor's

\footnotetext{
* Corresponding author. Tel.: +1-650-725-6827; fax: +1-650-723-1614.

E-mail address: webert@stanford.edu (T.A. Weber).
} 
actions to mitigate the risk of payment default. Garbling thus can provide a (possibly imperfect) substitute for missing contract dimensions such as ex-ante payment. ${ }^{1}$

Demski and Sappington (1986) provide an early application of strategic information garbling to influence agent action in a labor market delegation context, focusing on the timing of information release (rather than endogenizing its quality) and making simplifying assumptions about the allowable types of contracts. While limited liability has long been known to distort incentives in principal-agent situations (Fama and Miller, 1972; Sappington, 1983) and strategic information transmission has been shown to be generally useful in games (Aumann and Hart, 2003; Crawford and Sobel, 1982), information garbling has not, to our knowledge, been invoked as a contracting instrument to address the incentive distortion caused by limited liability.

\section{The model}

Consider a risk-averse investor with a twice differentiable Von Neumann-Morgenstern utility function $u$, so that $u^{\prime \prime}<0<u^{\prime}$. She can invest a portion $a \in[0, w]$ of her wealth $w$ in a risky asset of random return $\tilde{r} \in\left\{r_{1}, r_{2}\right\}$, where $r_{1} \in(-1,0)$ and $r_{2}>-r_{1}$. Initially the investor believes the return realizations are equally likely (uniform prior) and her utility maximization yields a unique optimal investment action of $a_{0}^{*}=\left[a_{0}^{u}\right]_{[0, w]}$, where $a_{0}^{u}$ is defined as the unique "unconstrained" solution of the first-order necessary optimality condition $E\left[\tilde{r} u^{\prime}\left(w+a_{0}^{u} \tilde{r}\right)\right]=0$. The "no-information" investment $a_{0}^{*}$ is always strictly greater than zero since the expected return from the risky asset is positive; it defines the investor's expected no-information utility $\bar{u}_{0}=E u\left(w+a_{0}^{*} \tilde{r}\right)$.

A risk-neutral information seller offers to sell the investor a signal $\tilde{s} \in\{L, H\}$, imperfectly correlated with $\tilde{r}$, where $\operatorname{Prob}(\tilde{s}=L)=\sigma_{L}, \operatorname{Prob}(\tilde{s}=H)=\sigma_{H}=1-\sigma_{L}$, and $\operatorname{Prob}\left(\tilde{s}=j \mid \tilde{r}=r_{i}\right)=q_{i j}$ for $i \in\{1,2\}$ and $j \in\{L, H\}$. If she were to purchase the signal, the investor would update her initial beliefs according to Bayes' rule and obtain the posterior return distribution $\pi_{i j}=\operatorname{Prob}\left(\tilde{r}=r_{i} \mid \tilde{s}=j\right)=q_{i j} /\left(2 \sigma_{j}\right)$. The posted price $p$ for the information is to be paid by the buyer after the return has been realized; its collection is subject to limited liability, in the sense that the information seller can at most claim all of the investor's remaining wealth. ${ }^{3}$ The investor's expected utility after purchasing the information, observing the signal realization $j \in\{L, H\}$ and choosing an investment level $a_{j} \in[0, w]$ is therefore

$$
\bar{u}_{j}\left(p, a_{j}\right)=\sum_{i} \pi_{i j} u\left(\left[w-p+a_{j} r_{i}\right]_{+}\right)
$$

1 Arrow (1962) (p. 111) pointed out that sellers of information needed to overcome both problems of adverse selection (buyers who fear they will receive low-quality information from sellers will not pay upfront) and moral hazard (buyers who, having had information revealed to them, refuse to pay), making both ex-ante and ex-post charges problematic. Ex-ante fees for information of unknown value are, in practice, uncommon, whereas ex-post charges reflective of the information value abound. Free trials of information products such as software are the norm; hedge funds and venture capital firms, who bundle private information with financial management services, typically charge $20 \%$ of ex-post profits ("carry") rather than up-front sales loads, and risk losing their carry if the portfolio's investments deteriorate. Recent work by Che and Gale (2000) indicates that partial ex-ante payments (which they term "cash bonds") can serve as a valuable signal of buyer wealth (allowing a more precise design of a pricing mechanism) rather than a method of conveying cash per se.

2 The $[\cdot]_{[0, w]}$ operator is defined for a given $w>0$ and any $\xi \in \mathbb{R}$ as $[\xi]_{[0, w]}=\max \{0, \min \{w, \xi\}\}$, truncating the value of $\xi$ to the admissible interval $[0, w]$.

3 In this model we suppose however that neither the investment actions a nor their results are directly contractible. 
where $[\xi]_{+}=\max \{0, \xi\}$ for any $\xi \in \mathbb{R}$. The information seller's revenue-maximization problem can now be written as

$$
\max _{p} \sum_{i, j} \sigma_{j} \pi_{i j} \min \left\{p,\left[w+a_{j}^{*} r_{i}\right]_{+}\right\}
$$

subject to both the investor's individual-rationality constraint,

$$
\sigma_{L} \bar{u}_{L}\left(p, a_{L}^{*}\right)+\sigma_{H} \bar{u}_{H}\left(p, a_{H}^{*}\right) \geq \bar{u}_{0}
$$

and her incentive-compatibility constraint,

$$
a_{j}^{*} \in \underset{a_{j} \in[0, w]}{\arg \max } \bar{u}_{j}\left(p, a_{j}\right) .
$$

The denominations of the signal realizations $L$ ("bad news") and $H$ ("good news") are chosen such that $a_{L}^{*} \leq a_{H}^{*}$. The investor's optimal informed investment in each state is obtained from (4), $a_{j}^{*} \in\left\{\left[a_{j}^{u}\right]_{[0, w]}, w\right\}$, where $a_{j}^{u}$ is defined analogously to $a_{0}^{u}$ as the interior optimum. The optimal investment $a_{j}^{*}$ is thus equal to either the amount $a_{j}^{u}$ determined by the first-order condition for maximizing $\bar{u}_{j}$ (truncated to the interval $[0, w]$ ) or her full wealth, $w^{4}{ }^{4}$ The investor prefers the latter, i.e. she "goes for broke", if the expected gain from increasing investment to the maximum possible is sufficiently "protected" by her limited payment liability in the case of a low return realization (Fama and Miller, 1972): the non-concavity of the investor's utility at the origin, introduced by limited liability, renders a threshold investment policy optimal (Gollier et al., 1997). If the no-information utility $\bar{u}_{0}$ is small enough, the seller can choose $p$ satisfying (3) such that the investor chooses an action in the "good news" signal state $H$ which leads to default for the negative return realization $r_{1}$. The prospect of such (partial) default through limited liability, escaping the consequences of a low return realization, implies that the investor will invest all her remaining wealth in the risky asset, $a_{H}^{*}=w$. Thus, the investor takes full advantage of the unsecured "loan" granted by the information seller through payment deferral. In stark contrast to this full commitment, the information seller would prefer the investor not to invest at all whenever her choice implies a risk of default on the ex-post payment.

\section{Garbling may increase revenue}

We now argue that to deter the investor from going for broke, the seller can distort the information by garbling his signal, mis-reporting its realizations. Instead of reporting both states $L$ and $H$ truthfully, the information seller can misreport realizations $L$ with probability $\alpha$ as $H$, whereas realizations $H$ are always reported truthfully. The new optimization problem with $\alpha$-garbling includes the original

\footnotetext{
${ }^{4}$ By the maximum theorem (Berge, 1959) the maximizer $a_{j}^{*}$ is upper hemicontinuous as a function of $p$. Indeed at the point of "switch" between interior investment $a_{j}^{u}$ and full investment $w$, the maximizer is in fact a set containing both $a_{j}^{u}$ and $w$ as elements.
} 
problem as a special case (for $\alpha=0$ ). The resulting probabilities $\hat{\sigma}_{j}$ of the signal reports observed by the investor are

$$
\begin{gathered}
\hat{\sigma}_{H}(\alpha)=\sigma_{H}+\alpha \sigma_{L}, \\
\hat{\sigma}_{L}(\alpha)=(1-\alpha) \sigma_{L} .
\end{gathered}
$$

In an $\alpha$-garbling environment the investor adjusts her posterior beliefs given a report of $H$ to

$$
\hat{\pi}_{i H}(\alpha)=\frac{\sigma_{H} \pi_{i H}+\alpha \sigma_{L} \pi_{i L}}{\sigma_{H}+\alpha \sigma_{L}}
$$

whereas given a report of $L$ her beliefs are not influenced by the garbling, $\hat{\pi}_{i L}=\pi_{i L}$. Since the $\alpha$-garbling affects the investor only following a report of "good news" and $\pi_{2 H}^{\prime}(\alpha) \leq 0$, it produces a posterior return distribution that is dominated, in the sense of first-order stochastic dominance, by the undistorted posterior distribution at $\alpha=0$ leading the investor to (weakly) lower investment actions and thereby decreasing her propensity to go for broke.

Consider the interesting case where $\hat{a}_{H}^{u}(0)<w$. The price $\hat{p}(\alpha)$ at which the investor is indifferent between investing an interior amount $\hat{a}_{H}^{u}(\alpha)$ and all her wealth, $w$, is implicitly defined by ${ }^{5}$

$$
\hat{u}_{H}\left(\hat{p}(\alpha), \hat{a}_{j}^{u}(\alpha) ; \alpha\right)=\hat{u}_{H}(\hat{p}(\alpha), w ; \alpha)
$$

where for $j \in\{L, H\}$ the signal-contingent expected utility $\hat{u}_{j}\left(p, a_{j} ; \alpha\right)$ is determined as in Eq. (1) with $\pi_{i j}$ and $\sigma_{j}$ replaced by $\hat{\pi}_{i j}(\alpha)$ and $\hat{\sigma}(\alpha)$ respectively. Similarly, $\hat{a}_{j}^{u}(\alpha)$ is determined in analogy to $a_{j}^{u}$, so that $E\left[\tilde{r} u^{\prime}\left(w-p+\hat{a}_{j}^{u}(\alpha) \tilde{r}\right)\right]=0$. We denote by $\bar{p}$ the expected revenue from posting the maximal individually rational price (without garbling). In order for an $\alpha$-garbling to yield a revenue improvement for the seller we require that ${ }^{6}$

$$
\hat{p}(\alpha) \geq \bar{p}
$$

The price $\hat{p}(\alpha)$ is thereby individually rational for the investor, if

$$
\hat{u}(\hat{p}(\alpha) ; \alpha) \geq \bar{u}_{0}
$$

where $\hat{u}(\hat{p}(\alpha) ; \alpha)=\hat{\sigma}_{L}(\alpha) \hat{u}_{L}\left(\hat{p}, \hat{a}_{L}^{*}(\alpha) ; \alpha\right)+\hat{\sigma}_{H}(\alpha) \hat{u}_{H}\left(\hat{p}, \hat{a}_{H}^{*}(\alpha) ; \alpha\right)$. The main result of the paper, that a revenue-improving garbling exists under these conditions, follows by construction.

5 In the event of "bad news" there may also exist a price at which the investor is indifferent between going for broke and choosing an interior maximizer. Going for broke in the low signal state, however, implies $a=\mathrm{w}$ in the high signal state as well. At any underlying non-negative price the individual rationality constraint cannot be satisfied, since a signal that induces the same action in all its states is inherently worthless, even under limited liability.

${ }^{6}$ If $\hat{p}(0)<\bar{p}$, then a simple price decrease may not deter the investor from going for broke and a minimum garbling $\alpha_{m}>0$ may be needed to yield any possible increase in the seller' revenue. If $\bar{p}<w\left(1+r_{1}\right)$, then the investor cannot default and has no incentive to go for broke, in which case $\bar{p}$ corresponds to the seller's optimal revenue. 
Proposition 1. (i) A revenue-increasing garbling exists, if there is an $\alpha^{*} \in(0,1)$ such that the relations (5)-(7) are satisfied. (ii) The $\alpha^{*}$-garbling is optimal, i.e. maximizes the information seller's revenue over all $\alpha \in[0,1]$, if in addition to (5) and (6) the constraint (7) is binding.

Proof. Part (i) follows directly from (5)-(7). Consider now part (ii). The information seller's revenue, $\sum_{i, j} \sigma_{j} \pi_{i j} \min \left\{p,\left[w+\hat{a}_{j}^{*} r_{i}\right]_{+}\right\}$, is non-decreasing in $p$ along (5). It is, therefore, sufficient to show that the maximum price, $\hat{p}$, at which the investor does not go for broke, i.e. which satisfies (5), is increasing in $\alpha$. Indeed if $\hat{p}(\alpha)$ is increasing, the largest $\alpha$ that satisfies both (5) and (7) is optimal. At this $\alpha^{*}$ the constraint (7) must be binding. From (5) we obtain using the envelope theorem:

$$
\hat{p}^{\prime}=-\frac{\sum_{i} \hat{\pi}_{i H}^{\prime}\left[u\left(\left[w-p+\hat{a}_{H}^{u} r_{i}\right]_{+}\right)-u\left(\left[w-p+w r_{i}\right]_{+}\right)\right]}{\hat{\pi}_{1 H} \partial_{p} u\left(\left[w-p+\hat{a}_{H}^{u} r_{1}\right]_{+}\right)+\hat{\pi}_{2 H} \partial_{p} u\left(\left[w-p+\hat{a}_{H}^{u} r_{2}\right]_{+}\right)} .
$$

The denominator of (8) is clearly negative, whereas the numerator is non-negative as a consequence of $\hat{\pi}_{2 H}^{\prime}<0<\hat{\pi}_{1 H}^{\prime}$ and the difference in square brackets is of the same sign as $\hat{\pi}_{i H}^{\prime}$ for all $i \in\{1,2\}$. Hence $\hat{p}^{\prime}>0$, which completes the proof.

\subsection{Numerical example}

Consider an investor with constant absolute risk aversion $\rho$ and utility function $u(w)=-\exp (-\rho w)$, who has initial wealth $w$ and has the option to invest in the return gamble $\tilde{r}$. Let $\left(w, \rho, r_{1}, r_{2}\right)=(1,2 / 3,-1$, 6). Assume that for all $j \in\{L, H\}$ we have $\sigma_{j}=0.5, q_{1 L}=0.8, q_{1 H}=0.2, q_{2 j}=1-q_{1 j}$. Using these parameters we find that the posted price making the investor's individual-rationality constraint (7)

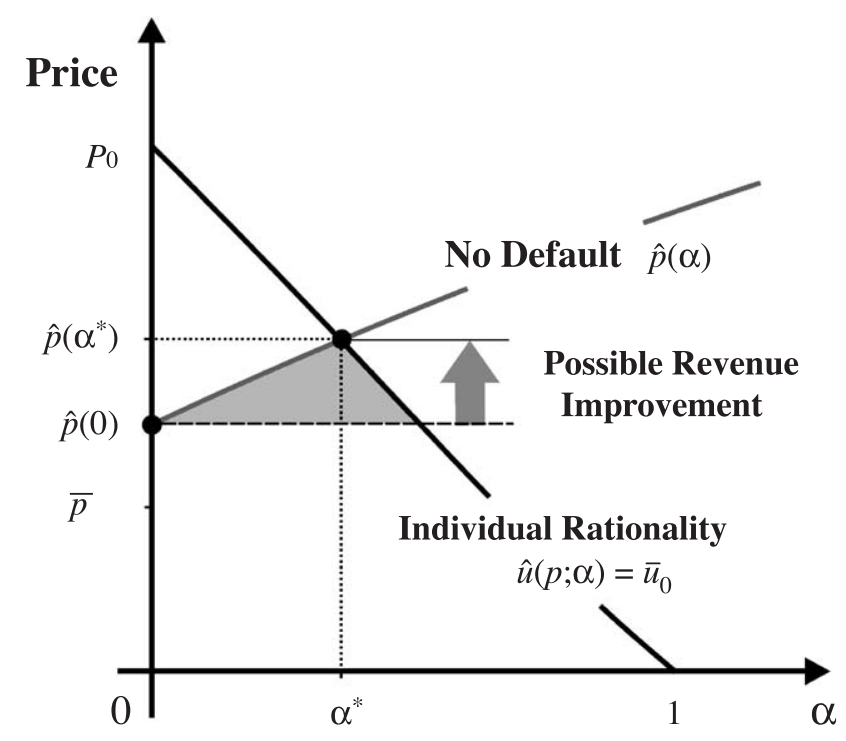

Fig. 1. Revenue-increasing garbling of information. 
binding is $p_{0}=0.161$, which leads to default in state $H$ when $r_{1}$ occurs. Hence the seller's expected revenue $\bar{p}=0.145$ is given by the right-hand side of (6) and is more than $10 \%$ smaller than the posted price $p_{0}$. Under $\alpha=0$ (no garbling), the amount which can be charged to guarantee zero default is equal to $\hat{p}(0)=0.149$, a certain revenue which is strictly larger than $\bar{p}$ while satisfying (5) and (7). Since $\hat{p}(\alpha)$ is increasing in $\alpha$, the seller can, through garbling, increase his revenues by removing the slack from (7) without inducing default. By Proposition 1 there exists a strictly revenue-increasing $\alpha$-garbling (cf. Fig. 1). The optimal garbling $\alpha^{*}=0.014$ makes (7) binding, generating a default-free certain revenue of $\hat{p}\left(\alpha^{*}\right)=0.155$ which is more than a $4 \%$ gain over $\hat{p}(0)$.

Note that, in our example, moving from $(0, \hat{p}(0))$ to $\left(\alpha^{*}, \hat{p}\left(\alpha^{*}\right)\right)$ is not a Pareto improvement, as the investor's individual-rationality constraint was slack at $\hat{p}(0)$ and is binding at $\hat{p}\left(\alpha^{*}\right)$. If however, $\hat{p}(0)<\bar{p}<\hat{p}\left(\alpha^{*}\right)$, then the optimal $\alpha^{*}$-garbling in the sense of Proposition 1 achieves a weak Pareto improvement, as the investor's individual-rationality constraint is binding for $\alpha \in\left\{0\right.$, $\left.\alpha^{*}\right\}$ (i.e. she is indifferent to the garbling) and the seller's revenue is strictly higher at $\alpha^{*}>0$ than at $\alpha=0$.

\section{Discussion}

Garbling a signal can be beneficial if an information seller can, by eliminating the investor's propensity to overinvest, thereby increase the probability of collecting her payment. In that sense, the realizable value of the signal can be non-monotonic in its informativeness as delivered. The deliberate damaging of the seller's information good through garbling eliminates the investor's moral hazard induced by the limited liability for the ex-post payment.

This benefit may outweigh the decrease in the investor's willingness to pay for a less informative signal. Our finding that delivering a lower-quality information product leads to higher revenues resonates with the similarly counter-intuitive negative marginal value of information noted by Cowen and Glazer (1996), in which less monitoring leads to higher effort in an agency context, as well as Demski and Sappington (1986) in which potentially useful information is deliberately withheld until after an agent has made a decision.

We have shown that garbling can be useful for an information seller in combination with a purely expost payment option. ${ }^{7}$ Naturally, increasing the number of revenue-extraction instruments cannot decrease revenues, but the result that a seller can achieve a strict revenue improvement by damaging his one and only information product may still come as a surprise.

\section{Acknowledgements}

This research was in part supported by the Risk Management and Decision Processes Center at the Wharton School of the University of Pennsylvania and by a David Morgenthaler II Faculty Scholar Award at Stanford University. We would like to thank Rachel Croson, Richard Kihlstrom, Paul Kleindorfer, an anonymous referee, and participants of the 2002 Conference on Economic Design in

\footnotetext{
7 It can be shown that, for signals of sufficiently high fidelity, purely ex-post payments may be (at least locally) optimal so that positive ex-ante payments would not be desirable even if explicitly permitted.
} 
New York City and the 2003 Spring Meeting of Young Economists in Leuven (Belgium) for helpful suggestions.

\section{References}

Arrow, K.J., 1962. Economic welfare and the allocation of resources for invention. The Rate and Direction of Inventive Activity. Princeton University Press, Princeton, NJ, pp. 609-625. Reprinted in Arrow, K.J., Collected Papers of Kenneth J. Arrow, vol. 5, Belknap Press, Cambridge, MA, pp. 104-119.

Aumann, R.J., Hart, S., 2003. Long cheap talk. Econometrica 71 (6), 1619-1660.

Berge, C., 1959. Espaces Topologiques et Fonctions Multivoques. Dunod, Paris.

Blackwell, D., 1953. Equivalent comparison of experiments. Annals of Mathematical Statistics 24 (2), $265-272$.

Che, Y., Gale, I., 2000. The optimal mechanism for selling to a budget-constrained buyer. Journal of Economic Theory 92 (2), 198-233.

Cowen, T., Glazer, A., 1996. More monitoring can induce less effort. Journal of Economic Behavior and Organization 30 (1), $113-123$.

Crawford, V.P., Sobel, J., 1982. Strategic information transmission. Econometrica 50 (6), 1431-1451.

Demski, J., Sappington, D., 1986. On the timing of information release. Information Economics and Policy 2 (4), $307-316$.

Fama, E., Miller, M., 1972. The Theory of Finance Dryden Press, Hinsdale, IL.

Gollier, C., Koehl, P.-F., Rochet, J.-C., 1997. Risk-taking behavior with limited liability and risk aversion. Journal of Risk and Insurance 64 (2), 347-370.

Marschak, J., Miyasawa, K., 1968. Economic comparability of information systems. International Economic Review 9 (2), $137-173$.

Sappington, D., 1983. Limited liability contracts between principal and agent. Journal of Economic Theory 29 (1), 1-21. 